\title{
Effectiveness and cost-effectiveness of guided Internet- and mobile-based CBT for adolescents and young adults with chronic somatic conditions and comorbid depression and anxiety symptoms (youthCOACH $\mathrm{CD}_{\mathrm{CD}}$ ): study protocol for a multicentre randomized controlled trial
}

Frederike Lunkenheimer ${ }^{1}$, Matthias Domhardt', Agnes Geirhos', Reinhold Kilian², Annabel S. Mueller-Stierlin², Reinhard W. Holl ${ }^{3}$, Thomas Meissner ${ }^{4}$, Kirsten Minden ${ }^{5,6}$, Morten Moshagen", Ramona Ranz ${ }^{3}$, Cedric Sachser ${ }^{8}$, Doris Staab ${ }^{5}$, Petra Warschburger ${ }^{9}$, Harald Baumeister ${ }^{1 *}$ and $\mathrm{COACH}$ consortium

\begin{abstract}
Background: Adolescents and young adults (AYA) with chronic somatic conditions have an increased risk of comorbid depression and anxiety symptoms. Internet- and mobile-based cognitive behavioural therapy (iCBT) might be one possibility to extend the access to evidence-based treatments. Studies suggest that guided iCBT can reduce anxiety and depression symptoms in AYA. However, little is known about the effectiveness of iCBT for AYA with chronic somatic conditions and comorbid symptoms of anxiety and/or depression in routine care. Evidence on the (cost-)effectiveness of iCBT is essential for its implementation in health care.

Objectives and methods: This multicentre two-armed randomized controlled trial (RCT) aims to evaluate the (cost-) effectiveness of guided iCBT (youthCOACH $\mathrm{CD}_{\mathrm{CD}}$ ) in addition to treatment as usual (TAU) compared to enhanced treatment as usual (TAU+) in AYA aged 12-21 years with one of three chronic somatic conditions (type 1 diabetes, cystic fibrosis, or juvenile idiopathic arthritis). AYA with one of the chronic somatic conditions and elevated symptoms of anxiety or depression (Patient Health Questionnaire [PHQ-9] and/or Generalized Anxiety Disorder [GAD-7] Screener score $\geq 7)$ will be eligible for inclusion. We will recruit 212 patients $(2 \times n=106)$ in routine care through three German patient registries. Assessments will take place at baseline and at 6 weeks, 3 months, 6 months, and 12 months postrandomization. The primary outcome will be combined depression and anxiety symptom severity as measured with the PHQ Anxiety and Depression Scale. Secondary outcomes will include health-related quality of life, coping strategies, self-efficacy, stress-related personal growth, social support, behavioural activation, adjustment and trauma-related symptoms, automatic thoughts, intervention satisfaction, working alliance, and Internet usage. The cost-effectiveness will be determined, and potential moderators and mediators of intervention effects will be explored.

(Continued on next page)
\end{abstract}

\footnotetext{
* Correspondence: harald.baumeister@uni-ulm.de

'Department of Clinical Psychology and Psychotherapy, Faculty of

Engineering, Computer Science and Psychology, Institute of Psychology and

Education, Ulm University, Albert-Einstein-Allee 47, 89081 Ulm, Germany

Full list of author information is available at the end of the article
}

(c) The Author(s). 2020 Open Access This article is distributed under the terms of the Creative Commons Attribution 4.0 International License (http://creativecommons.org/licenses/by/4.0/), which permits unrestricted use, distribution, and reproduction in any medium, provided you give appropriate credit to the original author(s) and the source, provide a link to the Creative Commons license, and indicate if changes were made. The Creative Commons Public Domain Dedication waiver (http://creativecommons.org/publicdomain/zero/1.0/) applies to the data made available in this article, unless otherwise stated. 
(Continued from previous page)

Discussion: iCBT might implicate novel ways to increase the access to evidence-based interventions in this specific population. The distinct focus on effectiveness and cost-effectiveness of youthCOACH $\mathrm{CD}_{\mathrm{C}}$ in patients with chronic somatic conditions, as well as intervention safety, will most likely provide important new insights in the field of paediatric e-mental health. A particular strength of the present study is its implementation directly into routine collaborative health care. As such, this study will provide important insights for health care policy and stakeholders and indicate how iCBT can be integrated into existing health care systems.

Trial registration: German Clinical Trials Register (DRKS), DRKS00017161. Registered on 17 September 2019.

Keywords: Depression, Anxiety, AYA, Internet- and mobile-based interventions, Chronic somatic conditions, RCT

\section{Background}

On average, $15 \%$ of children and adolescents suffer from chronic somatic conditions, and the trend is rising [1]. Disabling conditions are-amongst others-diabetes, with 310/100,000 children and adolescents [2], cystic fibrosis, with 8/100,000 [3], and juvenile idiopathic arthritis, which affects about 100/100,000 [4] individuals at the transition to adulthood. Current studies estimate the average annual social/economic costs of cystic fibrosis to be $€ 53,256$ (SD 46,589) [5], juvenile idiopathic arthritis $€ 27,634$ (SD 28,008) [6], and type 1 diabetes with $€ 3745$ (inter-quartile range 1943-4881) [7] in adolescents and young adults (AYA) per patient.

Mental disturbances and disorders are common in this population, particularly depression- and anxiety- related symptoms $[8,9]$, which in turn are associated with reduced quality of life, reduced treatment adherence, poorer long-term prognosis [10], and increased health service use (outpatient and inpatient, medical/medical claims, and pharmacy claims) [11].

AYA with chronic somatic conditions are confronted with two challenges simultaneously: disease-specific requirements (e.g. medication, visits to a physician, physical limitations, etc.) and age-specific developmental tasks (e.g. development of identity and autonomy) [9]. Hence, a significant part of AYA with chronic somatic conditions are in need of mental health support [12].

Cognitive behavioural therapy (CBT) has been shown to be efficacious in the treatment of depressive disorders $[13,14]$ and anxiety disorders $[15,16]$, and can be regarded as first-line treatment in children and adolescents [17]. With regard to symptoms of depression and anxiety in chronic somatic conditions, CBT has also been found to reduce symptoms of depressive disorders in adults with chronic somatic conditions [18-20], while the evidence on the effectiveness of CBT in AYA with somatic-mental health comorbidities is limited. Moreover, the access to treatments is limited. In a German sample, less than $30 \%$ of AYA with relevant mental health disturbances used mental health care offers (consulted a psychologist, psychiatrist, or psychotherapist) in the past 12 months [21]. Especially amongst AYA with chronic somatic conditions, the high prevalence rates of mental health problems combined with the very low treatment utilization rate show that methods to identify and disseminate empirically validated treatments of these disorders in health care are necessary [22, 23].

Internet- and mobile-based cognitive behavioural therapy (iCBT) might be a promising approach to overcome this mental health care gap and allow for a timely treatment of comorbid mental burden. Advantages of Internetand mobile-based interventions include temporal and local flexibility, widespread accessibility, presumed costeffectiveness, and low-threshold access to address psychological aspects of chronic conditions [24, 25]. iCBT, designed as self-help interventions with no or only very limited therapeutic guidance, can improve depressive symptoms and symptoms of various anxiety disorders in adults [26-29]. Several recent systematic reviews and meta-analyses indicate that iCBT is particularly efficacious when provided as therapeutically guided iCBT [30, 31]. As such, Internet- and mobile-based, therapeutically guided CBT can be as efficacious as face-to-face CBT for the treatment of mental disorders [32]. Thereby, iCBT might not only be efficacious but also safe and cost-effective. A recent meta-analysis of individual participant data showed that guided iCBT for adults with depression is associated with a mean reduced risk for symptom deterioration compared to other control conditions [33]. The results of a systematic review on cost-effectiveness indicate that guided Internet- and mobile-based interventions for the treatment of depression symptoms have the potential to be cost-effective [24]. This has also been shown for anxiety symptoms [34]. Also in this complex subpopulation of patients with somatic conditions, a systematic review reported that computerized CBT could also improve symptoms of anxiety and depression [35]. Another metaanalysis reported small to large effect sizes of iCBT on depression $(g=-.20)$, anxiety symptoms $(g=-.21)$, and physical health symptoms $(g=-1.14)$ within adults with somatic conditions [36]. Internet- and mobile-based interventions can also be used for the (co-)treatment of chronic somatic conditions, e.g. to increase diseasespecific self-efficacy in patients with diabetes $(d=.23)$ or 
improve pain-related disability in patients with chronic pain $[37,38]$.

When it comes to iCBT for AYA, the evidence is far less comprehensive. Still, current evidence suggests that iCBT can be efficacious in the treatment of anxiety and depression in AYA, again probably with higher effect sizes for guided iCBT compared to completely unguided self-help iCBT [31, 39]. However, whether this general conclusion holds true for the subpopulation of AYA with chronic somatic conditions is largely unknown; there are only a few small efficacy trials with some methodological flaws (e.g. high risk of bias due to inadequate blinding, lack of published protocols, incomplete outcome data) [40]. Thus, the effectiveness and cost-effectiveness of embedding iCBT for depression and anxiety in routine collaborative paediatric somatic care still need to be established.

\section{Methods/design Objectives}

This trial aims to evaluate the effectiveness and costeffectiveness of youthCOACH $\mathrm{CD}_{\mathrm{CD}}$, a guided iCBT for AYA with type 1 diabetes, cystic fibrosis, or juvenile idiopathic arthritis.

The primary objective is:

1) To evaluate the short-term effectiveness of $\mathrm{iCBT}$ in improving depression and anxiety symptoms, assessed using the Patient Health Questionnaire Anxiety and Depression Scale (PHQ-ADS), at 12 weeks post-randomization (t2) compared to enhanced treatment as usual (TAU+).

Secondary objectives of the study are:

2) To evaluate the middle (t3; 6 months postrandomization) and long-term ( $\mathrm{t} 4 ; 12$ months postrandomization) effectiveness of $\mathrm{iCBT}$ in improving depression and anxiety symptoms compared to TAU+.

3) To evaluate the short (t2), middle (t3), and longterm (t4) effectiveness of iCBT compared to TAU+ in improving (a) health-related quality of life, (b) coping strategies, (c) self-efficacy, (d) stress-related growth, (e) social support, (f) behavioural activation, (g) adjustment and trauma-focused symptoms, and (h) automatic thoughts and also to evaluate the (i) working alliance and (j) intervention satisfaction in the intervention group.

4) To examine the cost-effectiveness of iCBT compared to TAU+ over a period of 12 months.

5) To evaluate the safety of iCBT by examining potential adverse events compared to TAU+.

6) To explore potential moderators and mediators of intervention effects.

\section{Study design}

This is a two-armed, multicentre parallel randomized controlled trial (RCT) comparing iCBT to TAU+. The intervention group receives $\mathrm{iCBT}$ in addition to treatment as usual (TAU). Assessments will take place starting with mental health screening in paediatric units followed by five assessment points over one year: baseline (t0), 6 weeks ( $\mathrm{t} 1$ ), 3 months (t2), 6 months (t3), and 12 months (t4) post-randomization. The flow chart of the study design is shown in Fig. 1.

This clinical trial has been approved by the Ethics Committee of Ulm University (number 292/18) and will be conducted and reported in accordance with Consolidated Standards of Reporting Trials (CONSORT) statements for RCTs $[41,42]$ as well as the guidelines for executing and reporting Internet intervention research [43]. This trial will be monitored by an independent Data and Safety Monitoring Board (DSMB; CK, LK, CPS) and has been carefully checked for data protection compliance. This study protocol is in accordance with the Standard Protocol Items: Recommendations for Interventional Trials (SPIRIT) guideline [44]. The SPIRIT checklist is provided as Additional file 1.

\section{Participants and procedure Inclusion and exclusion criteria}

AYA between 12 and 21 years of age with type 1 diabetes, cystic fibrosis, or juvenile idiopathic arthritis and elevated anxiety and/or depression symptoms (Generalized Anxiety Disorder Screener, GAD-7 [45] and/or PHQ-9 [46] score $\geq 7$ ) with Internet access and a basic knowledge of the German language are eligible for inclusion. AYA with increased risk of suicidality at screening (PHQ-9 Item 9>1) will be excluded for ethical and safety reasons and will receive adequate care within the ongoing clinical routine. Prior to participation, consent to study participation must be given by the AYA or, for adolescents under the age of 16 , by a person with custody. As this is a pragmatic RCT, there are no further exclusion criteria.

\section{Recruitment}

Recruitment start is scheduled for October 2019. The iCBT with its associated independence of space and time enables a recruitment strategy throughout Germany. Anxiety and depression will be screened as part of the clinical routine in hospitals, clinics, medical practices, and medical centres across Germany where AYA with type 1 diabetes, cystic fibrosis, or juvenile idiopathic arthritis receive medical treatment. These clinical institutions are all organized within three well-established German patient registries, the National Paediatric Rheumatologic Database (NPRD) [47], the National Diabetes Registry (DPV) [48], and the 


\section{Recruitment}

AYA (12 - 21 years) with diagnosis of cystic fibrosis, juvenile idiopathic arthritis or type 1 diabetes and inclusion in the CF registry, NPRD or DPV

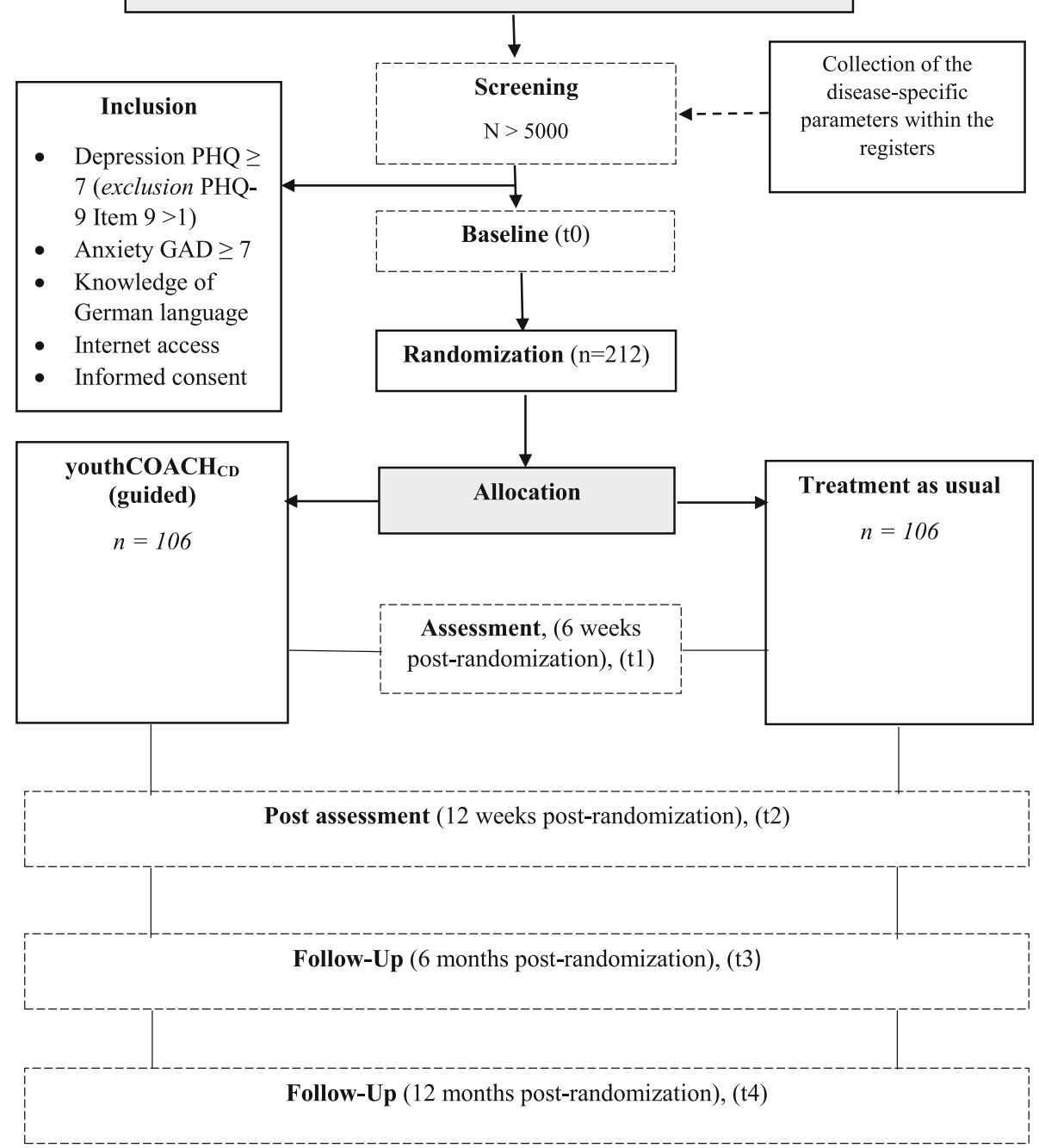

Note AYA $=$ Adolescents and young adults; $\mathrm{CF}$ registry $=$ Cystic Fibrosis Registry; NPRD $=$ National Paediatric Rheumatologic Database; DPV = National Diabetes Registry.

Fig. 1 Flow chart of inclusion and study procedure (youthCOACH $\mathrm{CD}=$ Internet- and mobile-based cognitive behavioural therapy)

Cystic Fibrosis (CF) Registry [49]. The screening data will be gathered in clinical centres and administered within these patient registries. AYA receive feedback on their mental well-being by their health care provider in charge. If AYA show anxiety and/or depression symptoms (GAD7 [45] and/or PHQ-9 [46] score $\geq 7$ ) without elevated suicidality (PHQ-9 Item 9 $\leq 1$ ), the respective patient is informed by the treating physician at the clinical centre and invited to participate in the study in addition to receiving standard care/TAU. The informed consent will be obtained at the clinic by the attending physician, and all further recruitment activities will be conducted by researchers at Ulm University. For each randomized AYA the respective clinical unit receives $€ 230$ as financial compensation for its recruitment effort. Additional study information is provided on https://coach.klips-ulm.de.

\section{Randomization}

The participants are randomized by the program Sealed Envelope (www.sealedenvelope.com) in an allocation ratio of 1:1 using block randomization. The block size is 6,8 , 10 participants per block and stratified according to the three chronic somatic conditions. Stratification is performed due to the strongly varying prevalence of chronic 
Table 1 Intervention content, based on cognitive behavioural therapy (CBT)

\begin{tabular}{|c|c|c|}
\hline & Content & \\
\hline Introduction: Welcome! & $\begin{array}{l}\text { Dealing with online training } \\
\text { Schedule, structure, and content } \\
\text { of online training } \\
\text { Introduction of the e-Coach }\end{array}$ & \\
\hline 1. Get to know your strengths! & $\begin{array}{l}\text { Creating goals } \\
\text { Becoming aware of strengths and } \\
\text { abilities } \\
\text { Use of own traits } \\
\text { Techniques to build robust self-esteem } \\
\text { Dealing with challenges/learning } \\
\text { problem-solving skills }\end{array}$ & Mood diary \\
\hline 2. Become active! & $\begin{array}{l}\text { Psychoeducational information on the } \\
\text { relationship between mood and behaviour } \\
\text { Exploring activities that give pleasure } \\
\text { Daily structuring to reduce stress } \\
\text { Integration of physical activity into daily life }\end{array}$ & \\
\hline 3. Overcoming fears & $\begin{array}{l}\text { Psychoeducational information on anxiety } \\
\text { Reduction of anxiety }\end{array}$ & \\
\hline 4. Learn to deal with bad moods & $\begin{array}{l}\text { Psychoeducational information on depression } \\
\text { Recognition and handling of rumination and } \\
\text { dysfunctional thoughts } \\
\text { Activation }\end{array}$ & \\
\hline $\begin{array}{l}\text { 5. Recognize and understand very } \\
\text { strong feelings! }\end{array}$ & $\begin{array}{l}\text { Psychoeducational information on emotions } \\
\text { Acceptance of emotions } \\
\text { Dealing with very strong feelings (e.g. skills, } \\
\text { relaxation techniques) }\end{array}$ & \\
\hline 6. Together we are stronger! & $\begin{array}{l}\text { Social competence training } \\
\text { Communication skills } \\
\text { Perception of social support } \\
\text { Increasing personal responsibility }\end{array}$ & \\
\hline 7. You did it! & $\begin{array}{l}\text { Summary of the learned contents } \\
\text { Coping strategies and support } \\
\text { Information on further treatment options } \\
\text { regarding mental health }\end{array}$ & \\
\hline
\end{tabular}

somatic conditions. Only RR, who is not involved otherwise in the trial process, will conduct the randomization process. AG, FL (study administration), and MM (statistical evaluation) are blinded and do not receive any information about the group allocation of study participants. The blinded members of the study team do not have access to documents showing group membership of participants.

\section{Study interventions \\ Intervention condition}

The intervention group receives the iCBT plus treatment as usual (TAU) and is compared to a control group, which receives $\mathrm{TAU}+$. youthCOACH $\mathrm{CD}_{\mathrm{CD}}$, developed by the Department of Clinical Psychology and Psychotherapy (FL, AG, MD, HB) consists of an introductory session with information about the online-based intervention and seven modules of approximately 50 to $70 \mathrm{~min}$ processing time each. Modules can be repeated as often as desired. The modules cover the topics motivation and resources, behavioural activation, understanding and coping with anxiety and depression, emotion regulation, communication, and social support as well as relapse prevention. The intervention is presented via information texts, videos, audio recordings, photos, metaphors, and therapeutic homework. The content of the intervention is based on CBT principles for depression and anxiety, including elements of psychoeducation, individual resources of the AYA, active coping (problem solving), restructuring of stressful disease-related thoughts, communication training, relaxation, and behaviour activation to model adaptive coping strategies.

The content of the intervention (see Table 1) is developed for AYA and related to typical challenges and tasks of a life with a chronic somatic condition. The intervention is explained and presented in a youth-friendly manner. To improve patient adherence, interactive elements (e.g. conditional contents, certificates) are implemented, and reminders of the weekly intervention lessons as well as homework assignments are forwarded via a mobile app. The theoretical skills learned in youthCOACH $\mathrm{CD}_{\mathrm{CD}}$ can be tested in practice and transferred into everyday life through homework (e.g. diaries), also via a mobile app. At the beginning of the intervention, participants are informed to receive daily 
Table 2 Intervention structure, technical implementations, and support

Intervention structure
- Seven weekly modules (50-70 min.)
Implemented elements
- Guidance by trained and supervised e-Coaches (graduates of a Mas-
ter's Degree, who are enrolled in a training program in CBT with chil-
dren and adolescents)
- One feedback message per module by e-Coaches
- Reminders by the e-Coach if the modules are not processed at a
date set in advance by the participant to promote adherence
- Deactivation and reactivation when modules are not processed and
three unsuccessful reminders
- Answers to additional questions from e-Coaches and the study team
- Tight security procedure for suicidal clues
- Weekly challenges/ homework
- Diaries via a mobile app with daily reminders
- Information given by text and videos
- Audio guided exercises
- Patient examples written and presented as audio recordings
- Metaphors, quizzes and conditional content
- Modules can be repeated as often as desired

reinforcing prompts via a mobile app during the intervention period. The reminders are sent automatically after each module has been completed and coordinated with intervention content to transfer the learned skills into everyday life of the participants. The mobile-based reminders aim to increase motivation of participants, remind participants to complete homework assignments, and repeat intervention content. The software solutions for the iCBT intervention and the mobile app are both provided by Minddistrict (www.minddistrict.com). Access to the iCBT intervention and the app is granted through an individual usernamepassword combination and will be available on a 24/7 basis.

\section{Guidance}

The intervention is therapist-guided by e-Coaches, who report semi-standardized feedback after each completed module on the intervention platform to the participant using an e-Coach manual. The e-Coach manual is standardized to guarantee protocol adherence by the eCoaches. e-Coaches are graduates of a Master's Degree in Psychology or Pedagogy, who are enrolled in a training program in CBT with children and adolescents, closely supervised by licensed psychotherapists. The feedback content matches the participants' tasks and is aimed to support adherence to treatment. The feedback also includes positive reinforcement to motivate participants to continue the intervention. For further questions, participants and e-Coaches can communicate via the intervention platform. e-Coaches will also send reminders to participants who do not complete intervention modules at the scheduled time via e-mail and telephone. The weekly schedule for processing a module is set by the participants themselves at the end of the previous module. In order to promote commitment, the intervention is blocked for the participant after three reminders without the participant reacting. For participants who indicate that they wish to continue, youthCOACH $\mathrm{CD}_{\mathrm{CD}}$ will be reactivated. Table 2 shows the intervention structure and implementation.

\section{TAU+}

TAU+ includes all routine care services for somatic and mental health problems. TAU+ can vary depending on the clinic, location, care options, and barriers of care as well as the specific needs of AYA. TAU is supplemented (enhanced, +) by screening for symptoms of anxiety and depression and by information letters on evidence-based mental health care options.

TAU+ is not defined a priori, but is recorded alongside the health economic evaluation and can thus be described post hoc descriptively.

\section{Sample size/power calculation}

A clinically significant effect of at least $d=.50$ for the mean difference between the groups is assumed, which refers to the severity of the primary outcome, i.e. a PHQ-ADS sum score [50] at 12 weeks postrandomization (t2). The assumption for the clinically significant effect is based on a meta-analysis by Ebert and colleagues, who reported a moderate to high effect size of $g=.72 \quad(95 \%$ confidence interval [CI] .55-.90) for Internet and computer-based CBT in the reduction of depressive and anxiety symptoms in youth [39], based on trials comparing Internet- and computer-based CBT to non-active control conditions (waitlist; placebo). However, given that TAU usually results in higher effect sizes compared to waitlist control groups, we regard a lower than $g=.72$ effect size as clinically relevant. The sample size calculation was carried out by an external statistician from the Department of Psychological Research Methods at Ulm University (MM) based on a power of .90 and $\alpha<.05$ (two tailed) considering the cluster structure (intraclass correlation $[\mathrm{ICC}]=.02$ ) of the different prevalence numbers of these chronic somatic conditions, resulting in 166 participants equally randomized to both conditions $(2 \times n=83)$. According to a metaanalysis, studies evaluating the efficacy and effectiveness of computer-based psychological treatments for depression with therapeutic support reported a dropout rate of approximately 28\% [51]. For this reason, the sample is increased by $28 \%$, resulting in a total of $N=212$ participants $(2 \times n=106)$. 
Various measures will be taken to minimize study drop-outs. Participants receive $€ 10$ as an expense allowance at all psychometric assessment points. After successful completion of all assessments, participants can also take part in a prize draw. Moreover, in order to avoid drop-outs in the intervention group, youth$\mathrm{COACH}_{\mathrm{CD}}$ was designed to persuade the target group of AYA using e.g. multimedia features such as videos and audios as well as prompts and reminders [52].

\section{Assessments and outcomes \\ Data collection methods}

The data from screening, outcome measurements, and economic measurements are collected from patients (patient-reported outcome) and from their caregivers/adult reference persons in tablet questionnaires (screening) or in online questionnaires. Medical record data of the participants are collected by physicians and are administered via the patient registers. The data is linked by a patient code (ID patient) and a hash key, in order to combine the data sources in a pseudonymized manner. The research data are stored on a protected cloud using encryption software. Detailed study management protocols and lists are used to monitor data collection. This allows interruptions and protocol deviations to be detected.

For an overview of instruments at screening, baseline ( $\mathrm{t} 0), 6$ weeks ( $\mathrm{t} 1), 3$ months ( $\mathrm{t} 2), 6$ months ( $\mathrm{t} 3$ ), and 12 months post-randomization (t4), see Table 3 .

\section{Inclusion criteria and outcome assessments}

Depression and/or anxiety symptom severity As part of clinical routine, AYA are screened for symptoms of depression and anxiety. The screening questionnaires are the GAD-7 [45] and the PHQ-9 [46] with a defined cut-off score of $\geq 7$ in either of the questionnaires [53].

The German version of the GAD-7 is a self-report anxiety questionnaire. It consists of seven items on a 4point scale and can be scored from $0=$ "not at all" to 3 $=$ "nearly every day". Good internal consistency $(\alpha=.79-.91)[54]$ and successful usage in adolescents are reported [55].

The German version of the PHQ-9 is administered as a screening inventory to detect depressive symptoms. The PHQ-9 consists of nine items on a 4-point scale with a rating scale ranging from $0=$ "not at all" to $3=$ "nearly every day". The computerized version $(\alpha=.88)$ of the PHQ-9 shows an equally high internal consistency as the paper-pencil version $(\alpha=.89)$ [56].

Sociodemographic variables As demographic data, we collect date of birth, gender, relationship status, type of school, grade of school or vocational training, and occupation.
Medical variables The collection of medical data takes place at the time of screening at the respective clinical centre. If the relevant parameters were not collected for screening, the closest examination is used at the time of screening; if two examinations are equally far apart, the previous examination is used, maximum one year away from screening. The following medical data of the participants are collected in the disease-specific registers depending on the chronic somatic condition:

1. Type 1 diabetes. HbA1c, number of severe hypoglycaemia (with and without coma) and diabetic ketoacidosis (DKA) events, continuous subcutaneous insulin infusion (CSII)/intensified conventional therapy (ICT), insulin dose per day, self-monitoring of blood glucose (SMBG), continuous glucose monitoring (CGM)/flash glucose monitoring (FGM), diabetes education, and celiac disease

2. Cystic fibrosis. Forced expiratory volume in 1 second (FEV 1), diabetes, pancreatic status, liver cirrhosis, pseudomonas infection, number of exacerbations, number of antibiotic therapies, and allergic bronchopulmonary aspergillosis (ABPA)

3. Juvenile idiopathic arthritis. Physician's global assessment of disease activity (numeric rating scale [NRS] 0-10) [57], active joint count, patient/parent global assessment of overall well-being (NRS), the clinical 10-joint Juvenile Arthritis Disease Activity Score [58], functional status (assessed by the Childhood Health Assessment Questionnaire) [59], Creactive protein $(\mathrm{CrP})$ and juvenile idiopathic arthritis category (systemic arthritis, persistent oligoarthritis, extended oligoarthritis, negative rheumatoid factor polyarthritis, positive rheumatoid factor polyarthritis, enthesitis-related arthritis, psoriatic arthritis, other arthritis).

\section{Outcome measurements}

Primary outcome: depressive and anxiety symptom severity The primary outcome is the combined depressive and anxiety symptom severity at $\mathrm{t} 2$, assessed with the PHQADS [50]. Depression and anxiety symptoms at all other assessments will be considered as secondary outcomes. PHQ-ADS is the combined sum score of the questionnaires GAD-7 and PHQ-9 as a composite measure of depression and anxiety, with good internal consistency $(\alpha=.88$ to .92$)[50]$.

\section{Secondary outcomes}

Coping with chronic health conditions The Coping with a Disease (CODI) questionnaire for children and adolescents with chronic health conditions [60] is a selfreport questionnaire to assess coping strategies of 
Table 3 SPIRIT schedule of enrolment, intervention, and assessments

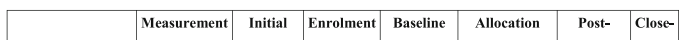

\begin{tabular}{|l|c|c|c|c|c|c|c|c|c|} 
& Measurement & $\begin{array}{c}\text { Initial } \\
\text { Screening }\end{array}$ & Enrolment & $\begin{array}{c}\text { Baseline } \\
\text { assessment }\end{array}$ & Allocation & \multicolumn{2}{|c|}{$\begin{array}{c}\text { Post- } \\
\text { Allocation }\end{array}$} & $\begin{array}{c}\text { Close- } \\
\text { Out }\end{array}$ \\
\hline TIMEPOINT & & & & $t 0$ & & \multicolumn{1}{c|}{$t 1$} & $t 2$ & $t 3$ & $t 4$ \\
\hline
\end{tabular}

\section{ENROLMENT:}

Eligibility screen

Depression and/or

anxiety sympton

severity

Not suicidal

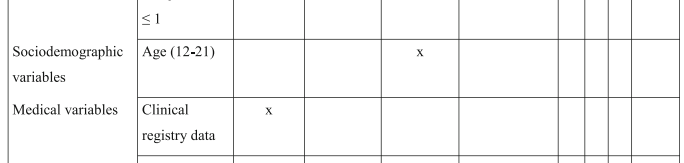

Informed consent

Allocation

INTERVENTION:

Intervention group

Control group

ASSESSMENTS:

Primary outcome

Depression and

severity

Secondary

outcomes

Depression and

anxiety symptom

severity

Coping

Self-Efficacy

Traumatic

experiences

Stress-related

Stross-re
growth

Social support

Intervention

satisfaction

Therapeutic allianc

Behavioural

Activation

Automatic thoughts

Quality of life

Caregiver Reports

Anxiety symptom

severity (caregiver)

Depression

symptom severity

(caregiver)

Social support

(caregiver)

Traumatic

experience

(caregiver)

Adverse events

Subjective side

effects

Internet usage

behaviour

Suicidal ideations

Symptom

Symptom

Deterioratio

Economic

evaluation

medical and

medical and

psychosocis services

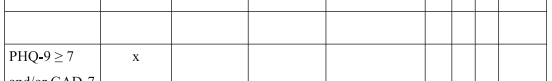

PQ- $9 \geq 7$

$>7$

tata

\begin{tabular}{|l|l|l|l|l|l|l|l|}
\hline & & & & $x$ & & & \\
\hline
\end{tabular}
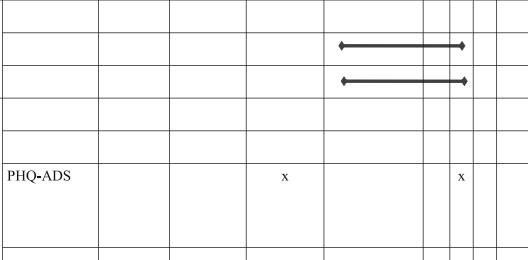

PHQ-ADS

PHQ-ADS
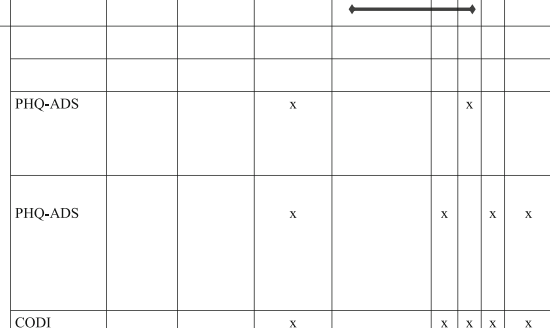

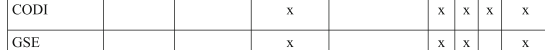
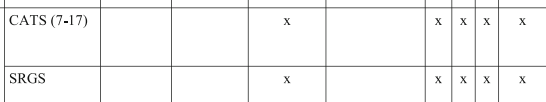

BSSS

CSQ-I

WAIS-R

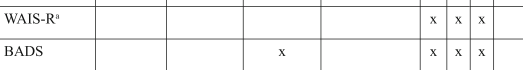

BADS

EQ-5D-Y

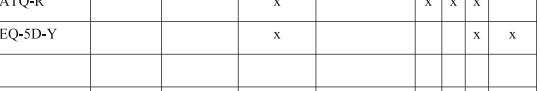

SCARED

MFQ

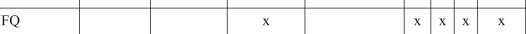

BS
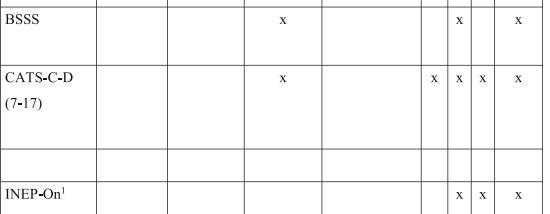

IUES

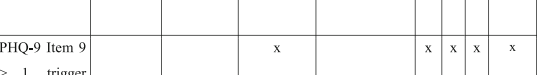

$>1$, trigger

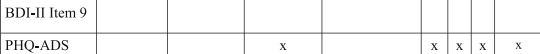

DE

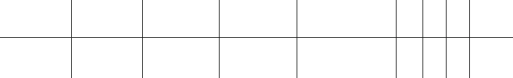

ISHI-

RI-

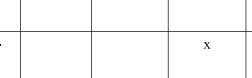

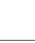

Notes: $\mathrm{t} 0=$ baseline, $\mathrm{t} 1=6$ weeks, $\mathrm{t} 2=3$ months, $\mathrm{t} 3=6$ months, $\mathrm{t} 4=12$ months, PHQ-9 Patient Health Questionnaire, GAD-7 Generalized Anxiety Disorder

Questionnaire, $P H Q-A D S$ Patient Health Questionnaire Anxiety and Depression

Scale, CODI Coping with a Disease, GSE General Self-Efficacy Scale, CATS (7-17) Child and Adolescent Trauma Screen, SRGS Stress-Related Growth Scale,

BSSS Berlin Social Support Scales, subscale "Actually Received Support", CSQ-

$I$ Client Satisfaction Questionnaire adapted to Internet-based interventions,

WAI-SR Working Alliance Inventory-Short Revised, BADS Behavioral Activation

for Depression Scale, ATQ-R Automatic Thoughts Questionnaire-Revised, EQ-

$5 D-Y$ EuroQol Five-Dimensional Questionnaire-Youth, SCARED Screen for Child

Anxiety Related Emotional Disorders, MFQ Mood and Feelings Questionnaire-

Caregiver, CATS-C-D (7-17) Child and Adolescent Trauma Screen-Caregiver,

INEP-On Inventory for recording negative effects of online interventions,

IUES Internet-Use Expectancies Scale, BDI-II Beck Depression Inventory-Revised,

CAMHSRI-DE Child and Adolescent Mental Health Services Receipt Inventory

${ }^{\mathrm{a}}$ Recorded in intervention group only

children and adolescents (ages ranging from 8 to 18 years) with chronic health conditions. The CODI questionnaire includes 29 items on a 5-point scale with a rating scale ranging from $1=$ "never" to $4=$ "all the time". The questionnaire shows an internal consistency depending on the domain between $\alpha=.69$ and $\alpha=.83$ [60].

General perceived self-efficacy General perceived selfefficacy represents a personal coping resource with predictive value for well-being and a constructive coping with life [61, 62]. It serves the personal assessment of one's own competence, generally to cope with difficulties and barriers in daily life successfully. The reported psychometric parameters of the General Self-Efficacy Scale (GSE) are satisfactory. The internal consistency ranges from $\alpha=.80$ to .90 for various German samples [63]. The 10 items of the GSE can be rated on a 4-point scale from $1=$ "is not true" to $4=$ "is absolutely right".

Traumatic experiences and personal growth The symptom list of the Child and Adolescent Trauma Screen (CATS) for ages 7-17 years is used to observe the changes within treatment in psychological stress caused by traumatic events due to a chronic somatic condition [64]. The questions about trauma-specific symptoms are introduced by the request that the AYA refer to the currently most stressful event with regard to their chronic condition and describe it briefly. The severity of the symptom scale can be rated from $0=$ "never" to $3=$ "almost always". Limitations of the functional level in different areas of life are also assessed. In all three countries where the scale has been validated (the USA, Germany, Norway), the 20-item symptom score of the self-report proved good to excellent reliability between $\alpha=.88$ and $\alpha=.94$ [64].

The Stress-Related Growth Scale (SRGS) serves to observe the changes that occur during treatment in relation to the individual development caused by the chronic disease. The construction of the SRGS [65] followed the theoretical concept of coping resources of Schaefer and Moos [66]. The present study uses the 15- 
item short form $(\alpha=.89)$ adapted to the specific population of AYA with a chronic somatic disorder. The items are answered on a 3-point scale with the values $0=$ "not at all", 1 = "something", and 2 = "a lot" [67].

Social support in coping with the disease The Berlin Social Support Scales (BSSS) [68] differ from other questionnaire methods for social support by their multidimensional approach, that is, by their cognitive and behavioural aspects. AYA are asked whom they relate their answers to. The original questionnaire consists of six scales (Perceived, Received and Achieved Support, Need and Search for Support, Protective Buffering). In this study the subscale "Actually Received Support, Recipient" is used. The subscale includes 11 items plus a general score, and the four-step answer format ranges from "strongly disagree" to "strongly agree". The subscale together with the general score shows an internal consistency of $\alpha=.83$ [68].

Intervention satisfaction Participants' satisfaction with the Internet-based intervention will be assessed with the Client Satisfaction Questionnaire adapted to Internetbased interventions (CSQ-I) [69]. The self-report questionnaire consists of eight items that are rated on a 5point Likert scale from 1 = "does not apply to me" to 4 $=$ "does totally apply to me". The scale shows a very good reliability, indicated by McDonald $\omega$ values of .93 to .95 . The CSQ-I will be used during the intervention (t1 and t2), only for participants receiving youthCOACH $\mathrm{CD}_{\mathrm{CD}}$.

Therapeutic alliance In the present study, therapeutic alliance between client and e-Coach/the Internet-based intervention will be assessed with an adapted version of the Working Alliance Inventory-Short Revised (WAISR) questionnaire [70]. The 12-item self-report questionnaire is rated on a 5 -point Likert scale $(1=$ "rarely" to 5 = "all the time"). For the German version, internal consistencies between $\alpha=.81$ and $\alpha=.91$ were reported for the three subscales (Agreement on Tasks, Agreement on Goals, and Development of an Affective Bond) and an internal consistency between $\alpha=.90$ and $\alpha=.93$ for the total score [71]. The WAI-SR will be completed by the intervention group only.

Behavioural activation The Behavioral Activation for Depression Scale (BADS) [72] examines the role of controlling aversive stimuli and avoidance behaviour in depression. As such, it observes changes in activation within treatment. The BADS consists of 25 questions, each rated on a 7-point scale ranging from $0=$ "not at all" to $6=$ "completely". The total score demonstrated acceptable internal consistency $(\alpha=.79)$ [73].
Automatic thoughts The Automatic Thoughts Questionnaire-Revised (ATQ-R) [74] records the influence of the intervention on automatic thoughts. The German version of the questionnaire consists of 21 items and can be rated on a 5-point scale between "not at all" and "all the time" [75]. The internal consistencies of the scales of the German version are between $\alpha=.75$ and $\alpha=.89$. All scales showed significant correlations with depressiveness and differentiated between adolescents aged between 11 and 16 years with higher and lower depressive symptom severity [75].

Quality of life The generic EuroQol Five-Dimensional Questionnaire (EQ-5D), measuring quality of life, is a short instrument that provides information on health states as a basis for the estimation of quality-adjusted life years (QALYs) [76]. A version for respondents aged 8 to 18 years is the EQ-5D-Youth version (EQ-5D-Y) [77, 78], developed based on the standard EQ-5D [79]. The available utility value sets for adults have been found to be not applicable to children and adolescents [80]. In the absence of a utility value set for children and adolescents in Germany [77], the health states will be valued by means of the visual analogue scale (VAS) of the EQ-5DY. The EQ-5D-Y consists of five items and can be evaluated by a 3-point scale ("no problems", "some problems", "a lot of problems"). The EQ-5D-Y dimensions were found to be reliable on test-retest (in 69.8-93.8\% of Italian youths and in $86.2-99.7 \%$ of Spanish respondents) [79]. The EQ-5D-Y not only serves as a secondary outcome, but is also used for economic evaluation.

\section{Caregiver reports}

Depressive and anxiety symptom severity In order to be able to record the severity of depression and anxiety symptoms not only in self-judgment but also in caregiver reports, a caregiver/adult reference person of the participant is asked to rate the Screen for Child Anxiety Related Emotional Disorders (SCARED) [81] questionnaire and the Mood and Feelings Questionnaire (MFQ) [82].

The SCARED, which examines the third-party rating of anxiety symptoms of the last 3 months, consists of five items and is scored on a 3-point scale from "not true or rare" to "accurate or frequent". The scale has an acceptable to good internal consistency ( $\alpha=.74$ to $\alpha=.93$ ) $[83,84]$.

The MFQ parent report of depressive symptoms is useful both for preliminary screening and to monitor change in symptomatology [82]. The MFQ short version consists of 13 items, which are rated by a reference person on a 3-point scale from "not true" to "true". The 
internal consistency of the original scale is good, with $\alpha=.91$ to $\alpha=.96[85,86]$.

Social support The BSSS [68] will be answered by AYA participants (more information can be found in the section "Secondary outcomes") and caregivers. The BSSS caregiver report is used to identify how the caregivers assess their own social support for the adolescent. Internal consistency for the 11-item scale "Actually Received Support" is good, with $\alpha=.75$ [68].

Traumatic experiences Whether the treatment has an influence on the effects of traumatic experiences due to the chronic somatic condition is explored by the stress symptom list of the Child and Adolescent Trauma Screen-Caregiver (CATS-C-D) for AYA aged 7-17 years, from the point of view of the caregivers. The questions about trauma-specific symptoms are introduced by the request that the caregivers refer to the currently most stressful event for the respective AYA with regard to their chronic somatic condition and describe it briefly. The severity of the symptom scale can be rated from $0=$ "never" to $3=$ "almost always". The 20 -item symptom score of the observer report has proven good reliability with $\alpha=.87$. Limitations of the functional level in different areas of life are also assessed [64].

\section{Adverse events}

Subjective side effects Possible unwanted/negative effects of the intervention ( $t 2, \mathrm{t} 3$, and $\mathrm{t} 4$, only for participants receiving youthCOACH $\mathrm{CD}_{\mathrm{CD}}$ ) are assessed by the 23 item Inventory for Recording Negative Effects of Online Interventions (INEP-On). This is an adapted version of the Inventory for recording negative effects of psychotherapy that is specifically adjusted for online interventions [87]. Eleven items are rated on a 7-point bipolar scale ( $-3=$ "better; is totally true" to $+3=$ "worse; is totally true") and 12 items on a 4-point Likert scale $(0=$ "no agreement at all" to $3=$ "total agreement"). The original scale shows a high internal consistency of $\alpha=.85$ [87].

Symptom deterioration The PHQ-ADS [50] is not only used as a primary or secondary outcome, depending on the assessment time point, but also to determine depression and anxiety symptom deterioration.

Internet usage behaviour In order to explore the change in Internet usage due to treatment, the questionnaire Internet-Use Expectancies Scale (IUES) [88] is used. The IUES consists of eight items with a two-factor structure, that is positive and avoidance expectancies. The items can each be rated on a 6-point Likert scale.
Both factors have good reliability ("positive expectancies", Cronbach's $\alpha=.83$ and "avoidance expectancies", Cronbach's $\alpha=.76$ ) [88]. In addition to the questionnaire, open questions are asked about the duration of use for the Internet and the smartphone, either privately or educationally/professionally.

Suicidal ideations Notwithstanding that suicidality is defined as an exclusion criterion, suicidal ideation might occur during the course of the study and will therefore be monitored closely by means of the PHQ-9 Item 9 at all assessment points $(\mathrm{t} 0-\mathrm{t} 4)$. A score $\geq 1$ on the suicidality item of PHQ-9 ("Thoughts that you would be better off dead or of hurting yourself in some way?") leads to the Beck Depression Inventory-Revised (BDI-II) [54] suicidality item (Item 9; BDI-II item $=0$ : "I'm not thinking of harming myself.", BDI-II item = 1: "I have thoughts of killing myself, but I would not carry them out", BDI-II item $=2$ : "I would like to kill myself", BDI-II item $=3$ : "I would kill myself if I had the chance") [89]. A score $\geq 1$ on the BDI-II suicidality item results in a standardized suicide prevention protocol.

Participants screening positive (BDI-II Item $9 \geq 1$ ) receive an online information letter with detailed information on available and appropriate health services with the advice to use professional help. Participants are informed that youthCOACH $\mathrm{CD}_{\mathrm{CD}}$ is not optimized for suicidal ideation management.

In the case of BDI-II Item $9 \geq 2$, participants automatically receive, in addition to the information letter with detailed information on available and appropriate health services, an individual safety plan to fill out (numbers of personal contacts, counselling, medical on-call and emergency numbers), and the project team will follow a detailed, stepped suicide prevention protocol. These participants will be contacted by one of three licensed (child and adolescent) psychotherapists via telephone and interviewed in regard to suicidality. In case of acute suicidality, participants are directed to appropriate on-site mental health care services, when they are compliant. In case of acute suicidality and perceived non-compliance, contact details of participants will be immediately transferred to emergency services in order to secure the safety of participants and initiate appropriate crises intervention; non-compliant participants will be subsequently excluded from the trial. Participants who do not indicate acute suicidal ideations and express compliance will proceed with the study routine.

In order to counteract unexpected side effects or to be able to react when they occur, it is possible for the study participants to contact the study team during working hours by telephone or e-mail. Participants are referred to a nationwide and 24/7 emergency doctor's telephone hotline and receive detailed information about treatment 
options. If participants indicate suicidality, the abovementioned prevention protocol takes effect.

\section{Economic evaluation: use and costs of medical and psychosocial services}

The Child and Adolescent Mental Health Services Receipt Inventory (CAMHSRI-DE) will be applied to collect information on the clients' use of health and social services. The extent of service usage is determined on the basis of frequency and duration for eight service categories (inpatient medical services, outpatient medical services, social services-inpatient and outpatient, other inpatient services, school-based services, school types, and medication intake) [90]. The frequency and duration for each health care service will be interpolated for a reference period of 6 months. The CAMHSRI-DE is based on the Client Sociodemographic and Service Receipt Inventory (CSSRI) [91, 92], adjusted for children and adolescents with mental health problems in the German health care system [90]. Costs of service use will be estimated on the basis of unit cost information [93].

\section{Statistical analyses}

All statistical analyses are processed by a biostatistician who is blinded with regard to group assignment. Patterns of missing data will be examined. Multiple imputation with predictive mean matching will be performed to account for missing data. All analyses will be conducted on a two-sided level of significance $(\alpha=.05)$. Participant characteristics will be described descriptively. Rates of patientreported adverse events will be compared.

\section{Effectiveness analysis}

There will be no interim evaluation of the primary outcome. All statistical analyses will be performed based on the intention-to-treat (ITT) principle. Additional per protocol analyses will be conducted in order to examine the effects of youthCOACH $\mathrm{CD}_{\mathrm{CD}}$ in case of patients adhering to the intervention protocol. Participants who completed at least $80 \%$ of the intervention are defined as intervention completers (= per protocol).

The primary outcome will be analysed using a hierarchical linear model to account for clustering with the PHQ-ADS score at $\mathrm{t} 2$ as dependent variable and the baseline value as covariate, adjusting for sex, age, and chronic somatic condition. Standardized mean differences and 95\% CIs will be calculated to measure the between-group effect size at post-treatment (t2) and follow-up $(t 3, t 4)$. Secondary outcomes will be analysed accordingly.

Exploratory mediation and moderator analyses involving the primary and secondary outcomes as well as demographic data will be conducted. Moderator and subgroup analyses will be attempted in case of a sufficiently large sample size.

\section{Health economic evaluation}

Cost-effectiveness analyses of the $\mathrm{iCBT}$ intervention will be estimated from the societal perspective by means of the net benefit method [94-96]. The incremental costeffectiveness ratio (ICER) will be computed to estimate the maximum willingness to pay (MWTP) $\lambda$ necessary for the gain of one QALY by iCBT in comparison to TAU+. The stochastic uncertainty of the ICER will be estimated by non-parametric bootstrapping $[95,96]$. The interpretation of results is based on the costeffectiveness acceptance curve using a range of MWTP thresholds between 0 and 100,000 $€$ [95-97].

\section{Discussion}

The aim of this study is to examine the (cost-)effectiveness of youthCOACH $\mathrm{CD}_{\mathrm{CD}}$, an iCBT for AYA with a chronic somatic condition and comorbid depressive and/or anxiety symptoms.

The innovative aspects of the study are the following: (1) youthCOACH $\mathrm{CD}_{\mathrm{CD}}$ is implemented directly into routine medical care. As such, the present study will provide important insights for health care policy on how to integrate iCBT into our health care systems. (2) Studies show that AYA between the ages of 12 and 25 spend an average of $22 \mathrm{~h}$ a week online with an increasing tendency to communicate, play, or entertain. Four out of five of 12 - to 17 -year-olds $(80.6 \%)$ have used the Internet daily in the last 12 months [98]. Only 4.6\% of adolescents at this age do not make use of the Internet. The fact that AYA grow up as digital natives could make online use of an intervention well accepted.

Continuing with advantages, the methodical strengths of the study are as follows: (3) The area-wide recruitment via the German patient registers enables a representative sample and facilitates generalization. (4) With a target sample of 212 adolescents, the study will be high-powered $(1-\beta=.90)$. (5) In addition to self-report questionnaires, the study also includes caregiver selfreports on their perception of participants' conditions. This provides further insights into the effectiveness of iCBT. (6) Not only the effectiveness of youthCOACH $\mathrm{CD}_{\mathrm{CD}}$, but also undesirable potential adverse events will be investigated in detail in this study. (7) As one of as yet only a few clinical trials on psychological interventions for AYA with chronic somatic conditions and symptoms of depression and anxiety, the present RCT also comprises cost-effectiveness analyses. (8) Finally, in addition to the psychological parameters, medical data are collected, which are linked to each other and thus allow deeper insights into comorbidity. 
The study has several possible obstacles and limitations. First, adolescents aged 12 to 16 years need the informed consent of the custodial persons to participate in the study. This legal constraint might lead to a leftskewed age distribution of the study participants. By adjusting the covariate baseline to age, sex, and chronic somatic disease, this possible limitation is taken into account in the statistical analysis.

Second, intervention and study adherence might be limited in AYA, as has been reported before [99]. Therefore, a drop-out rate of $28 \%$ was established. As a counterstrategy we developed our intervention based on persuasive design aspects and provide it therapeutically guided-measures that are associated with improved intervention adherence [51, 100]. Additionally, participants receive a monetary compensation for their study efforts, which will likely increase study adherence.

Third, participant inclusion is based on depression/ anxiety symptoms and not on mental disorder status. The reason for this is that the assessments are based on participants' self-report, which is complemented by caregiver reports. Therefore, participants, caregivers, and physicians of the recruitment supporting clinics are informed that youthCOACH $\mathrm{CD}_{\mathrm{CD}}$ is designed as a (guided) self-help intervention that does not replace on-site mental health care according to existing guidelines in case of manifest mental disorders.

Fourth, given the nature of the present trial, participants need to go through several self-report assessments. This might bias effectiveness results in two ways. On the one hand, the assessments might lead to a self-selection of participants with only those being randomized who are determined enough to finalize a comprehensive baseline assessment. At the same time, reminders on the follow-up assessments might have an intervention adherence facilitating effect, reminding participants not only to conduct the assessments but to continue the intervention or reminding them e.g. of learned intervention aspects. On the other hand, the comprehensive assessment with several self-report questionnaires might have a negative effect on trial adherence, increasing attrition rates. These not yet well examined and understood research biases on intervention effects inherent to RCTs need to be taken into account when interpreting the coming effectiveness findings.

In conclusion, Internet- and mobile-based interventions might have the potential to augment health care services substantially, as they could be a temporal and local flexible, accessible, and cost-effective treatment alternative. AYA with chronic somatic diseases and comorbid anxious and/or depressive symptoms could be supported by iCBT to reduce mental disturbances. With iCBT as a low-threshold and low-intensity intervention, progress and chronification of mental disorders might be preventable at an early stage. The first results of youthCOACH $\mathrm{CD}_{\mathrm{CD}}$ are expected to be available in 2021 .

\section{Trial status}

Protocol version number 1.0 (submitted on 22/08/2019). Recruitment start is scheduled for October 2019 and will be expected to complete in April 2021.

\section{Supplementary information}

Supplementary information accompanies this paper at https://doi.org/10. 1186/s13063-019-4041-9.

Additional file 1. SPIRIT 2013 Checklist: Recommended items to address in a clinical trial protocol and related documents.

\section{Abbreviations}

ATQ-R: Automatic Thoughts Questionnaire-Revised; AYA: Adolescents and young adults; BADS: Behavioral Activation for Depression Scale; BDI-II: Beck Depression Inventory-Revised; BSSS: Berlin Social Support Scales; CAMHSRIDE: Child and Adolescent Mental Health Services Receipt Inventory; CATS: Child and Adolescent Trauma Screen; CBT: Cognitive behavioural therapy; COACH: Chronic Conditions in Adolescents: Implementation and Evaluation of Patient-centred Collaborative Healthcare; CODI: Coping with a Disease (questionnaire for children and adolescents with chronic health conditions); CSQ-I: Client Satisfaction Questionnaire adapted to Internetbased interventions; EQ-5D-Y: EuroQol Five-Dimensional QuestionnaireYouth; GAD-7: Generalized Anxiety Disorder Screener; GSE: General SelfEfficacy Scale; iCBT: Internet- and mobile-based cognitive behavioural therapy; ICC: Intra-class correlation; ICER: Incremental cost-effectiveness ratio; INEP-On: Inventory for recording negative effects of online interventions; ITT: Intention-to-treat; IUES: Internet-Use Expectancies Scale; MFQ: Mood and Feelings Questionnaire (caregiver); PHQ-9: Patient Health Questionnaire; SCARED: Screen for Child Anxiety Related Emotional Disorders (caregiver); SD: Standard Deviation; SRGS: Stress-Related Growth Scale; TAU+: Treatment as usual+; WAI-SR: Working Alliance Inventory-Short Revised;

youthCOACH $\mathrm{CD}_{\mathrm{C}}$ : youthCOACH chronic disease

\section{Acknowledgements}

We commemorate Prof. Dr. Lutz Goldbeck, who initiated the $\mathrm{COACH}$ project and passed away unexpectedly in October 2017. His enormous knowledge and experience in the field of research with children and adolescents with chronic conditions and comorbid mental disorders and the high level of commitment of his study team led to the improvement of care for this population.

We thank Thorsten Sukale for supporting the realization of the standardized prevention protocol. We thank Lisa Büchler, Luisa Rehder, and Angela Serian for their support in developing the intervention content.

\section{Publication of study results}

Study results will be published in peer-reviewed journals and will be presented at national and international conferences. General information on the project and the results will be published on the COACH homepage (https:// coach.klips-ulm.de).

If the study results indicate that the iCBT intervention youthCOACH $\mathrm{CD}_{\mathrm{D}}$ is effective in reducing anxiety and depression symptoms, efforts will be undertaken to facilitate the implementation of this intervention into routine health care

\section{Authors' contributions}

$\mathrm{RK}, \mathrm{RH}, \mathrm{TM}, \mathrm{KM}, \mathrm{DS}, \mathrm{PW}$, and $\mathrm{HB}$ initiated this study. All authors contributed to the design of the study. FL, MD, AG, and $H B$ are responsible for the design of the present iCBT trial. FL wrote the draft of this manuscript; MD, $A G, R K, A M, R H, T M, K M, R R, C S, D S, P W$, and HB provided revisions. All authors contributed to the further writing and approved the final version of the manuscript. 


\section{Funding}

This study is realized as part of the project "Chronic Conditions in Adolescents: Implementation and Evaluation of Patient-centred Collaborative Healthcare $(\mathrm{COACH})$ ", funded by the German Federal Ministry of Education and Research (Grant identification 01GL1740A and 01GL1740E). The NPRD is funded by AbbVie, Chugai, and Novartis.

\section{Availability of data and materials}

Individual participant data will be made available on request after deidentification beginning 12 months following article publication of the effectiveness and the cost-effectiveness paper. Data will be made available to researchers who provide a methodologically sound proposal, not already covered by others. Proposals should be directed to the corresponding author. Data requestors will need to sign a data access agreement. Provision of data is subject to data security regulations. Investigator support depends on available resources.

\section{Ethics approval and consent to participate}

The study will be conducted in accordance with the principles of Good Clinical Practice, the Declaration of Helsinki (https://www.wma.net/wpcontent/uploads/2016/11/DoH-Oct2008.pdf), and current ethical standards. Written informed consent is obtained from each participant. Depending on the age of the participant, informed consent from the legal representative or guardian will also be required. The central ethical approval of the Ethics Committee of UIm University was obtained (request number 292/18). The $\mathrm{COACH}$ consortium will be advised by an external Data and Safety Monitoring Board (DSMB) consisting of three researchers in their areas of expertise: Prof. Dr. Christine Knaevelsrud (CS), Free University of Berlin; PD Dr. Levente Kriston (LK), University Hospital Hamburg-Eppendorf; Prof. Dr. Claus Peter Schmitt (CPS), Heidelberg University Hospital.

There are no known risks or side effects of Internet- and mobile-based interventions to reduce depressive symptoms in adults [34]. However, in case of unexpected (serious) adverse events with inevitable protocol modifications during the conduction of the trial, the necessary changes will be notified to the Ethics Committee of UIm University and the DSMB.

\section{Consent for publication}

Not applicable.

\section{Competing interests}

$\mathrm{HB}$ is a consultant for different insurance companies, health care associations, and psychotherapy chambers and gives talks and workshops and conducts a third party-funded project on iCBT and the dissemination and implementation of iCBT. The remaining authors declare that they have no competing interests.

\section{Author details}

${ }^{1}$ Department of Clinical Psychology and Psychotherapy, Faculty of Engineering, Computer Science and Psychology, Institute of Psychology and Education, Ulm University, Albert-Einstein-Allee 47, 89081 Ulm, Germany. ${ }^{2}$ Department of Psychiatry and Psychotherapy II, BKH Günzburg, UIm University, Günzburg, Germany. ${ }^{3}$ Institute of Epidemiology and Medical Biometry, ZIBMT, UIm University, Ulm, Germany. ${ }^{4}$ Department of General Pediatrics, Neonatology and Pediatric Cardiology, University of Düsseldorf, Düsseldorf, Germany. ${ }^{5}$ Charité University Medicine Berlin, Berlin, Germany. ${ }^{6}$ German Rheumatism Research Centre, Berlin, Germany. ${ }^{7}$ Department of Psychological Research Methods, Institute of Psychology and Education, UIm University, Ulm, Germany. ${ }^{8}$ Clinic of Child and Adolescent Psychiatry/ Psychotherapy, University Hospital Ulm, Ulm, Germany. ${ }^{9}$ Department Psychology, Counselling Psychology, University of Potsdam, Potsdam, Germany.

Received: 11 September 2019 Accepted: 30 December 2019 Published online: 12 March 2020

\section{References}

1. Van Der Lee J, Mokkink L, Grootenhuis M, Heymans H, Offringa M. Definitions and measurement of a systematic review. JAMA. 2007;297(24): $2741-51$.
2. Diabetes DE. Deutscher Gesundheitsbericht Diabetes 2010. 2010. http:// www.diabetesde.org/system/files/documents/gesundheitsbericht_2010_ gesamt_28_10_2009.pdf.

3. Farrell PM. The prevalence of cystic fibrosis in the European Union. J Cyst Fibros. 2008;7(5):450-3.

4. Luque Ramos A, Hoffmann F, et al. Transition to adult rheumatology care is necessary to maintain DMARD therapy in young people with juvenile idiopathic arthritis. Semin Arthritis Rheum. 2017;47(2):269-75.

5. Chevreul K, Michel M, Brigham KB, López-Bastida J, Linertová R, OlivaMoreno J, et al. Social/economic costs and health-related quality of life in patients with cystic fibrosis in Europe. Eur J Heal Econ HEPAC Heal Econ Prev Care. 2016;17(1):7-18.

6. Kuhlmann A, Schmidt T, Treskova M, López-Bastida J, Linertová R, Oliva-Moreno J, et al. Social/economic costs and health-related quality of life in patients with juvenile idiopathic arthritis in Europe. Eur J Health Econ. 2016;17(1):79-87.

7. Bächle C, Icks A, Straßburger K, Flechtner-Mors M, Hungele A, Beyer P, et al. Direct diabetes-related costs in young patients with early-onset, long-lasting type 1 diabetes. PLoS One. 2013;8(8):e70567.

8. Pinquart $M$, Shen Y. Anxiety in children and adolescents with chronic physical illnesses: a meta-analysis. Acta Paediatr. 2011;100:1069-76.

9. Pinquart $M$, Shen $Y$. Behavior problems in children and adolescents with chronic physical illness: a meta-analysis. J Pediatr Psychol. 36(4):375-84 http:// ovidsp.ovid.com/ovidweb.cgi? T=JS\&CSC=Y\&NEWS=N\&PAGE=fulltext\&D= emed10\&AN=21088072\%5Cn, http://oxfordsfx.hosted.exlibrisgroup.com/ oxford?sid=OVID:embase\&id=pmid:21088072\&id=doi:10.1093/jpepsy/jsq104 \&issn=1465-735X\&isbn=\&volume $=36 \&$ issue $=4 \&$ spage $=375 \&$ page .

10. Fidika A, Herle M, Goldbeck L. Symptoms of depression impact the course of lung function in adolescents and adults with cystic fibrosis. BMC Pulm Med. 2014;14(1):1-6 http://www.pubmedcentral.nih.gov/articlerender.fcgi?tool= pmcentrez\&artid=4277836\&rendertype $=a b s t r a c t \% 5 C n$, http://www. pubmedcentral.nih.gov/articlerender.fcgi?tool=pmcentrez\&artid=4277836.

11. Janicke DM, Harman JS, Kelleher KJ, Zhang J. The association of psychiatric diagnoses, health service use, and expenditures in children with obesityrelated health conditions. J Pediatr Psychol. 2009;34(1):79-88.

12. De Ridder D, Geenen R, Kuijer R, Van Middendorp H. Review psychological adjustment to chronic disease; 2008. p. 372.

13. DeRubeis RJ, Siegle GJ, Hollon SD. Cognitive therapy vs. medications for depression: treatment outcomes and neural mechanisms. Nat Rev Neurosci. 2009;9(10):788-96

14. Deutsche Gesellschaft für Kinder- und Jugendpsychiatrie, Psychosomatik und Psychotherapie D. Behandlung von depressiven Störungen bei Kindern und Jugendlichen: evidenz- und konsensbasierte Leitlinie (S3): AWMF online-Das Portal der wissenschaftlichen Medizin; 2013. https://www.awmf. org/leitlinien/detail/II/028-043.html.

15. Bandelow B, Sher L, Bunevicius R, Hollander E, Kasper S, Zohar J, et al. Guidelines for the pharmacological treatment of anxiety disorders, obsessive-compulsive disorder and posttraumatic stress disorder in primary care. Int J Psychiatry Clin Pract. 2012;16(2):77-84.

16. Deutsche Gesellschaft für Kinder- und Jugendpsychiatrie, Psychosomatik und Psychotherapie D. Behandlung von Angststörungen bei Kindern und Jugendlichen: evidenz- und konsensbasierte Leitlinie (S3): AWMF online-Das Portal der wissenschaftlichen Medizin; 2010. https://www.awmf.org/ leitlinien/detail/I//051-028.html.

17. Compton SN, March JS, Brent D, Albano AM, Weersing VR, Curry J. Cognitive-behavioral psychotherapy for anxiety and depressive disorders in children and adolescents: an evidence-based medicine review. J Am Acad Child Adolesc Psychiatry. 2004;43(8):930-59.

18. Dobkin RD, Menza M, Allen LA, Gara MA, Mark MH, et al. Cognitivebehavioral therapy for depression in Parkinson's disease: a randomized, controlled trial. Am J Psychiatry. 2011;168:1066-74.

19. Baumeister $H$, Huttel N, Bengel J. Psychological and pharmacological interventions for depression in patients with coronary artery disease. Cochrane Database Syst Rev. 2011;9:CD008012 http://bf4dv7zn3u.search. serialssolutions.com.myaccess.library.utoronto.ca/?url_ver=Z39.88-2004\&rft_ val_fmt=info:ofi/fmt:kev:mtx:journal\&rfr_id=info:sid/Ovid:medl\&rft.genre= article\&rft_id=info:doi/10.1002\%2F14651858.CD008012.pub3\&rft_id=info: pmid/21901717.

20. Baumeister $\mathrm{H}$, Hutter N. Collaborative care for depression in medically ill patients. Curr Opin Psychiatry. 2012;25(5):405-14.

21. Hintzpeter B, Klasen F, Schön G, Voss C, Hölling H, Ravens-Sieberer U. Mental health care use among children and adolescents in Germany: results 
of the longitudinal BELLA study. Eur Child Adolesc Psychiatry. 2015;24(6): 705-13.

22. Chavira DA, Stein MB, Bailey K, Stein MT. Child anxiety in primary care: prevalent but untreated. Depress Anxiety. 2004;20(4):155-64.

23. Costello EJ, He J, Sampson NA, Kessler RC, Ries MK. Services for adolescents with psychiatric disorders: 12-month data from the National Comorbidity Survey-Adolescent. Psychiatr Serv. 2014;65(3):359-66.

24. Paganini S, Teigelkötter W, Buntrock C, Baumeister H. Economic evaluations of internet- and mobile.based interventions for the treatment and prevention of depression: a systematic review. J Affect Disord. 2018;225(1): 733-55.

25. Andersson G, Titov N. Advantages and limitations of Internet-based interventions for common mental disorders. World Psychiatry. 2014;13(1):4-11.

26. Hedman E, Ljótsson B, Lindefors N. Cognitive behavior therapy via the Internet: a systematic review of applications, clinical efficacy and costeffectiveness. Expert Rev Pharmacoecon Outcomes Res. 2012;12:745-64.

27. Hedman E, El Alaoui S, Lindefors N, Andersson E, Rück C, Ghaderi A, et al. Clinical effectiveness and cost-effectiveness of Internet- vs. group-based cognitive behavior therapy for social anxiety disorder: 4-year follow-up of a randomized trial. Behav Res Ther. 2014;59:20-9.

28. Domhardt $M$, Geßlein $H$, von Rezori R, Baumeister $H$. Internet-and mobilebased interventions for anxiety disorders: a meta-analytic review of intervention components. Depress Anxiety. 2019;36:213-24.

29. Königbauer J, Letsch J, Doebler P, Ebert D, Baumeister H. Internet- and mobile-based depression interventions for people with diagnosed depression: a systematic review and meta-analysis. J Affect Disord. 2017; 223(April):28-40.

30. Baumeister $H$, Reichler L, Munzinger $M$, Lin J. The impact of guidance on Internet-based mental health interventions - a systematic review. Internet Interv. 2014;1(4):205-15 https://doi.org/10.1016/j.invent.2014.08.003.

31. Domhardt M, Steubl L, Baumeister H. Internet- and mobile-based interventions for mental and somatic disorders in children and adolescents: a systematic review of meta-analyses. Z Kinder Jugendpsychiatr Psychother. 2020;48:33-46

32. Carlbring P, Andersson G, Cuijpers P, Riper H, Hedman-Lagerlöf E. Internet-based vs. face-to-face cognitive behavior therapy for psychiatric and somatic disorders: an updated systematic review and meta-analysis. Cogn Behav Ther. 2018;47(1):1-18 https://doi.org/10.1080/16506073.201 7.1401115 .

33. Ebert DD, Donkin L, Andersson G, Andrews G, Berger T, Carlbring P, et al. Does Internet-based guided-self-help for depression cause harm? An individual participant data meta-analysis on deterioration rates and its moderators in randomized controlled trials. Psychol Med. 2016;46(13):2679-93.

34. Dear BF, Zou JB, Ali S, Lorian CN, Johston L, Sheehan J, et al. Clinical and cost-effectiveness of therapist-guided internet-delivered cognitive behavior therapy for older adults with symptoms of anxiety: a randomized controlled trial. Behav Ther. 2015;46:206-17.

35. McCombie A, Gearry R, Andrews J, Mikocka-Walus A, Mulder R. Computerised cognitive behavioural therapy for psychological distress in patients with physical illnesses: a systematic review. J Clin Psychol Med Settings. 2015;22(1):20-44.

36. Farrand $\mathrm{P}$, Woodford J. Effectiveness of cognitive behavioural self-help for the treatment of depression and anxiety in people with long-term physical health conditions: a systematic review and meta-analysis of randomised controlled trials. Ann Behav Med. 2015:49(4):579-93.

37. Bendig E, Bauereiß N, Ebert DD, Snoek F, Andersson G, Baumeister $H$. Internet-based interventions in chronic somatic disease. Dtsch Aerztebl Online. 2018;(October):659-66 https://www.aerzteblatt.de/10.3238/arztebl.2 018.0659 .

38. Lin J, Paganini S, Sander L, Lüking M, Daniel Ebert D, Buhrman M, et al. Internetbasierte Intervention bei chronischen Schmerzen. Dtsch Arztebl Int. 2017;114(41):681-8.

39. Ebert DD, Zarski AC, Christensen H, Stikkelbroek Y, Cuijpers P, Berking M, et al. Internet and computer-based cognitive behavioral therapy for anxiety and depression in youth: a meta-analysis of randomized controlled outcome trials. PLoS One. 2015;10(3):1-15 https://doi.org/10.1371/journal. pone.0119895

40. Thabrew H, Stasiak K, Hetrick SE, Wong S, Huss JH, Merry SN. E-Health interventions for anxiety and depression in children and adolescents with long-term physical conditions. Cochrane Database Syst Rev. 2018;8: CD012489.
41. Moher D, Hopewell S, Schulz KF, Montori V, Gøtzsche PC, Devereaux PJ, et al. CONSORT 2010 explanation and elaboration: updated guidelines for reporting parallel group randomised trials. BMJ. 2010;340:C869.

42. Schulz KF, Altman DG, Moher D. CONSORT 2010 Statement: updated guidlines for reporting parallel group randomised trials. BMJ. 2010;340:c332.

43. Proudfoot J, Klein B, Barak A, Carlbring P, Cuijpers P, Lange A, et al. Establishing guidelines for executing and reporting internet intervention research. Cogn Behav Ther. 2011;40:82-97.

44. Chan A-W, Tetzlaff JM, Altman DG, Laupacis A, Gøtzsche PC, Krleža-Jerić K, Hróbjartsson A, Mann H, Dickersin K, Berlin JA, Doré CJ, Parulekar WR, Summerskill WSM, et al. SPIRIT 2013 Statement: Defining Standard Protocol Items for Clinical Trials. Ann Intern Med. 2013;158(3):200-7.

45. Spitzer RL, Kroenke K, Williams JBW, Löwe B. A brief measure for assessing generalized anxiety disorder. Arch Intern Med. 2006;166(10):1092 http:// archinte.jamanetwork.com/article.aspx?doi=10.1001/archinte.166.10.1092.

46. Richardson LP, McCauley E, Grossmann DC, McCarty CA, Richards J, Russo JE, Rockhill C, Katon W. Evaluation of the Patient Health Questionnaire-9 Item for detecting major depression among adolescents. Pediatrics. 2010; 126(6):1117-23.

47. Minden K, Niewerth M, Listing J, Zink A. German Study Group of Pediatric Rheumatologists. Health care provision in pediatric rheumatology in Germany--national rheumatologic database. J Rheumatol. 2002;29(3):622-8.

48. Hofer SE, Schwandt A, Holl RW, Austrian/German DPV Initiative. Standardized documentation in pediatric diabetology: experience from Austria and Germany. J Diabetes Sci Technol. 2016;10:1042-9.

49. Stern M. The use of a cystic fibrosis patient registry to assess outcomes and improve cystic fibrosis care in Germany. Curr Opin Pulm Med. 2011;17:473-7.

50. Kroenke K, Wu J, Yu Z, Bair MJ, Kean J, Stump T, et al. Patient health questionnaire anxiety and depression scale: initial validation in three clinical trials. Psychosom Med. 2016;78(6):716-27.

51. Richards D, Richardson T. Computer-based psychological treatments for depression: a systematic review and meta-analysis. Clin Psychol Rev. 2012; 32(4):329-42 https://doi.org/10.1016/j.cpr.2012.02.004.

52. Baumeister H, Kraft R, Baumel A, Pryss R, Messner E-M. Persuasive e-health design for behavior change. In: Baumeister H, Montag C, editors. Mobile sensing and digital phenotyping. Berlin: Springer; 2019.

53. Plummer F, Manea L, Trepel D, McMillan D. Screening for anxiety disorders with the GAD-7 and GAD-2: a systematic review and diagnostic metaanalysis. Gen Hosp Psychiatry. 2016;39:24-31 https://doi.org/10.1016/j. genhosppsych.2015.11.005.

54. Dear BF, Titov N, Sunderland M, McMillan D, Anderson T, Lorian C, Robinson E. Psychometric comparison of the Generalized Anxiety Disorder Scale-7 and the Penn State Worry Questionnaire for measuring response during treatment of generalised anxiety disorder. Cogn Behav Ther. 2011;40(3):216-27 https://doi.org/10.1080/16506073.2011.582138.

55. Puka K, Widjaja E, Lou SM. The influence of patient, caregiver, and family factors on symptoms of anxiety and depression in children and adolescents with intractable epilepsy. Epilepsy Behav. 2017;67:45-50 https://doi.org/10.1 016/j.yebeh.2016.12.011.

56. Erbe D, Eichert $H C$, Rietz C, Ebert D. Interformat reliability of the patient health questionnaire: validation of the computerized version of the PHQ-9. Internet Interv. 2016;5:1-4 https://doi.org/10.1016/j.invent.2016.06.006.

57. Filocamo G, Daví S, Pistorio A, Bertamino M, Puberto N, Lattanzi B, Consolaro A, Mahni-Manzoni S, Galasso R, Varnier GC, Martini A, Ravelli A. Evaluation of 21-numbered circle and 10-cm horizontal line visual analog scales for physician and parent subjective ratings in juvenile idiopathic arthritis. J Rheumatol. 2010;37(7):1534-41.

58. Consolaro A, Giancane G, Schiappapietra B, Davì S, Calandra S, Lanni S, et al. Clinical outcome measures in juvenile idiopathic arthritis. Pediatr Rheumatol. 2016;14(23):1-8 https://doi.org/10.1186/s12969-016-0085-5.

59. Singh G, Athreya B, Fries JF, Goldschmith DP. Measurement of health status in children with juvenile rheumatoid arthritis. Arthritis Rheum. 1994;37:1761-9.

60. Petersen C, Schmidt S, Bullinger M. DISABKIDS Group. Brief report: development and pilot testing of a coping questionnaire for children and adolescents with chronic health conditions. J Pediatr Psychol. 2004;29(8):635-40.

61. Jerusalem M. Persönliche Ressourcen, Vulnerabilität und Stresserleben. Göttingen: Hogrefe; 1990.

62. Schwarzer R. Optimistische Kompetenzerwartung: zur Erfassung einer personalen Bewältigungsressource. Diagnostica. 1994;40:105-23. 
63. Schumacher J, Klaiberg A, Brähler E. Bevölkerungsrepräsentative Normierung der Skala zur Allgemeinen Selbstwirksamkeitserwartung. Diagnostica. 2001; 15(31):1-8.

64. Sachser C, Berliner L, Holt T, Jensen TK, Jungbluth N, Risch E, et al. International development and psychometric properties of the Child and Adolescent Trauma Screen (CATS). J Affect Disord. 2017;210(August 2016): 189-95 https://doi.org/10.1016/j.jad.2016.12.040.

65. Park $\mathrm{CL}$, Cohen LH, Murch RL. Assessment and prediction of stress-related growth. J Pers. 1996;64(1):71-105 http://doi.wiley.com/10.1111/j.1467-6494.1 996.tb00815.x.

66. Schaefer JA, Moos GH. Life crises and personal growth. In: Carpenter BN, editor. Personal coping: theory, research and application. Westport: Praeger; 1992. p. 149-70

67. Maercker A, Langner R. Persönliche Reifung (personal growth) durch Belastungen und Traumata: Validierung zweier deutschsprachiger Fragebogenversionen. Diagnostica. 2001;47:153-62.

68. Schulz U, Schwarzer R. Soziale Unterstützung bei der Krankheitsbewältigung: die Berliner Social Support Skalen (BSSS). Diagnostica. 2003;49(2):73-82.

69. Boß L, Lehr D, Reis D, Vis C, Riper H, Berking M, Ebert DD. Reliability and validity of assessing user satisfaction with web-based health interventions. J Med Internet Res. 2016;18(8):e234.

70. Wilmers F, Munder T, Leonhart R, Herzog T, Plassmann R. Deutschsprachige Version des Working Alliance Inventory short revised (WAI-SR) - ein schulübergreifendes, ökonomisches und empirisch validiertes Instrument zur Erfassung der therapeutischen Allianz. Klin Diagnostik und Eval. 2008; 1(3):343-58

71. Munder T, Wilmers F, Leonhart R, Linster HW, Barth J. Working alliance inventory-short revised (WAI-SR): psychometric properties in outpatients and inpatients. Clin Psychol Psychother. 2010;17:231-9.

72. Teismann T, Ertle A, Furka N, Willutzki U, Hoyer J. The German version of the Behavioral Activation for Depression Scale (BADS): a psychometric and clinical investigation. Clin Psychol Psychother. 2016;23(3):217-25.

73. Kanter JW, Mulick PS, Busch AM, Berlin KS, Martell CR. The Behaviora Activation for Depression Scale (BADS): psychometric properties and factor structure. J Psychopathol Behav Assess. 2007;29(3):191-202.

74. Kendall C, Howard BL, Hays RC. Self-referent speech and psychopathology: the balance of positive and negative thinking. Cognit Ther Res. 1989;13: 583-98.

75. Huffziger S, Meyer TD, Seemann S, Horn AB, Groen G, Possel P. Evaluation of a German-language tool for measuring positive and negative automatic thoughts in children and adolescents. (Eval eines deutschsprachigen Instrumentes zur Erfassung positiver und negativer automische Gedanken bei Kindern und Jugendlichen). 2008;37(4):255-62. http://ovidsp.ovid.com/ ovidweb.cgi?T=JS\&PAGE=reference\&D=psyc6\&NEWS=N\&AN=2008-15862-005.

76. Szende A, Janssen B, Cabases J. Self-reported population health: an international perspective based on EQ-5D. Dordrecht: Springer Netherlands; 2014.

77. Kreimeier S, Greiner W. Themed Section: Evolution of EuroQoL. EQ-5D-Y as a health-related quality of life instrument for children and adolescents: the instrument's characteristics, development, current use, and challenges of developing its value set. Value Health. 2019;22(1):31-7 https://doi.org/10.1 016/j.jval.2018.11.001

78. Wille N, Badia X, Bonsel G, Burström K, Cavrini G, Devlin N, et al. Development of the EQ-5D-Y: a child-friendly version of the EQ-5D. Qual Life Res. 2010;19(6):875-86.

79. Ravens-Sieberer U, Wille N, Badia X, Bonsel G, Burström K, Cavrini G, et al. Feasibility, reliability, and validity of the EQ-5D-Y: results from a multinational study. Qual Life Res. 2010;19(6):887-97.

80. Craig BM, Greiner W, Brown DS, Reeve BB. Valuation of child health-related quality of life in the United States. Health Econ. 2016;777(April 2015):768-77.

81. Birmaher B, Khetarpal S, Brent D, Cully M, Balach L, Kaufman J, Mckenzie NS. The Screen for Child Anxiety Related Emotional Disorders (SCARED): scale construction and psychometric characteristics. J Am Acad Child Adolesc Psychiatry. 1997;36(4):545-53

82. Costello EJ, Angold A. Scales to assess child and adolescent depression: checklists, screens, and nets. J Am Acad Child Adolesc Psychiatry. 1988;27(6): 726-37

83. Essau CA, Muris P, Ederer EM. Reliability and validity of the Spence's Children's Anxiety Scale and the Screen for Anxiety Related Emotional Disorders in German children. J Behav Ther Exp Psychiatry. 2002;33:1-18.
84. Birmaher B, Brent D, Chiappetta L, Bridge J, Monga S, Baugher M. Psychometric properties of the Screen for Child Anxiety Related Emotional Disorders (SCARED): a replication study. J Am Acad Child Adolesc Psychiatry. 1999:38(10):1230-6.

85. Burleson DW, Birmaher B, Melhem NA, Axelson DA, Michaels SM, Brent DA. Criterion validity of the Mood and Feelings Questionnaire for depressive episodes in clinic and non-clinic subjects. J Child Psychol Psychiatry Allied Discip. 2006;47(9):927-34.

86. Thabrew H, Stasiak K, Bavin LM, Frampton C, Merry S. Validation of the Mood and Feelings Questionnaire (MFQ) and Short Mood and Feelings Questionnaire (SMFQ) in New Zealand help-seeking adolescents. Int J Methods Psychiatr Res. 2018;27(3):e1610.

87. Ladwig I, Rief W, Nestoriuc Y. Welche Risiken und Nebenwirkungen hat Psychotherapie? - Entwicklung des Inventars zur Erfassung Negativer Effekte von Psychotherapie (INEP). Verhaltenstherapie. 2014;24(4):252-63 https:// www.karger.com/?doi=10.1159/000367928.

88. Brand M, Laier C, Young KS. Internet addiction: coping styles, expectancies, and treatment implications. Front Psychol. 2014;5(Nov):1-14.

89. Kühner C, Bürger C, Keller F, Hautzinger M. Reliabilität und validität des revidierten Beck-Depressionsinventars (BDI-II). Befunde aus deutschsprachigen Stichproben. Nervenarzt. 2007;78(6):651-6.

90. Beecham J, Knapp M. Costing psychiatric services. In: Thornicroft G, editor. Measuring mental health. London: Gaskell; 2001. p. 200-24.

91. Chisholm D, Knapp MRJ, Knudsen HC, Amaddeo F, Gaite L. Client SocioDemographic and Service Receipt Inventory - European Version: development of an instrument for international research. Br J Psychiatry. 2000;177(39):28-33.

92. Roick C, Kilian R, Matschinger H, Bernert S, Mori C, Angermeyer MC. Die deutsche Version des Client Sociodemographic and Service Receipt Inventory. Psychatrische Prax. 2001;28(2):84-90.

93. Weschenfelder A-K, Reissner V, Knollmann M, Hebebrand J, Wasem J, Neumann A. Ökonomische Evaluation eines manualbasierten Therapiekonzeptes für psychisch kranke Kinder und Jugendliche mit schulvermeidendem Verhalten. Z Kinder Jugendpsychiatr Psychother. 2018; 46(1):47-56.

94. Glick HA. Economic evaluation in clinical trials. Reprinted. Oxford: Oxford University Press; 2010

95. Salize H-J, Kilian R. Gesundheitsökonomie in der Psychiatrie. Stuttgart: Kohlmann; 2010

96. Willan AR, Briggs AH. Statistical analysis of cost-effectiveness data. New York: Wiley; 2006.

97. Thokala P, Ochalek J, Leech AA, Tong T. Cost-effectiveness thresholds: the past, the present and the future. Pharmacoeconomics. 2018;36(5):509-22.

98. Orth B. Die Drogenaffinität Jugendlicher in der Bundesrepublik Deutschland 2015. Teilband Computerspiele und Internet. BZgA-Forschungsbericht. Köln: Bundeszentrale für gesundheitliche Aufklärung; 2017.

99. Reyes-Portillo JA, Mufson L, Greenhill LL, Gould MS, Fisher PW, Tarlow N, et al. Web-based interventions for youth internalizing problems: a systematic review. J Am Acad Child Adolesc Psychiatry. 2014;53(12):1254-1270.e5 https://doi.org/10.1016/j.jaac.2014.09.005.

100. Andersson G. Internet-delivered psychological treatments. Annu Rev Clin Psychol. 2016;12(1):157-79 http://www.annualreviews.org/doi/10.1146/ annurev-clinpsy-021815-093006.

\section{Publisher's Note}

Springer Nature remains neutral with regard to jurisdictional claims in published maps and institutional affiliations.

\section{Ready to submit your research? Choose BMC and benefit from:}

- fast, convenient online submission

- thorough peer review by experienced researchers in your field

- rapid publication on acceptance

- support for research data, including large and complex data types

- gold Open Access which fosters wider collaboration and increased citations

- maximum visibility for your research: over $100 \mathrm{M}$ website views per year

At $\mathrm{BMC}$, research is always in progress.

Learn more biomedcentral.com/submission 